TI 2015-015/VIII

Tinbergen Institute Discussion Paper
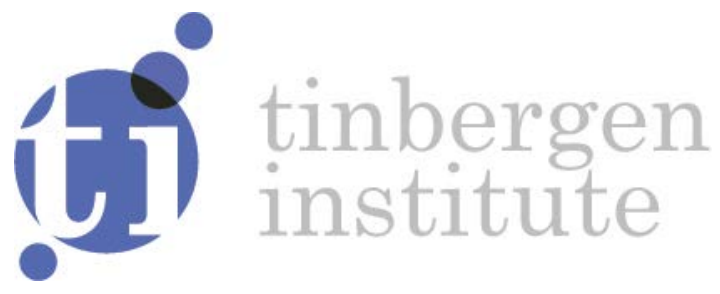

\title{
International Environmental Agreements with Support
}

REVI SI ON: J ULY 2017

Erik Ansink ${ }^{1}$

Hans-Peter Weikard ${ }^{2}$

Cees Withagen ${ }^{1}$

${ }^{1}$ Vrije Universiteit Amsterdam and Tinbergen Institute, The Netherlands

${ }^{2}$ Wageningen University, The Netherlands 
Tinbergen Institute is the graduate school and research institute in economics of Erasmus University Rotterdam, the University of Amsterdam and VU University Amsterdam.

Contact: discussionpapers@tinbergen.nl

More TI discussion papers can be downloaded at http://www.tinbergen.nl

Tinbergen Institute has two locations:

Tinbergen Institute Amsterdam

Gustav Mahlerplein 117

1082 MS Amsterdam

The Netherlands

Tel.: +31(0)205984580

Tinbergen Institute Rotterdam

Burg. Oudlaan 50

3062 PA Rotterdam

The Netherlands

Tel.: +31(0)10408 8900 


\title{
International environmental agreements with support*
}

\author{
Erik Ansink $^{\dagger} \quad$ Hans-Peter Weikard $\quad$ Cees Withagen ${ }^{\S}$ \\ July 4, 2017
}

\begin{abstract}
We augment the standard cartel formation game from non-cooperative coalition theory, often applied in the context of international environmental agreements, with the possibility that singletons support coalition formation without becoming coalition members themselves. We assume their support takes the form of a monetary transfer to the coalition, in order to induce larger coalitions, higher levels of public good provision and higher payoffs. We show that, under mild conditions on the costs and benefits of contributing to the public good (e.g. abatement of greenhouse gas emissions), there exist equilibria with support. Allowing for support increases payoffs to each of three types of agents: members, supporters and free-riders.
\end{abstract}

Keywords: Coalition formation; Public goods; Support; Transfers; International Environmental Agreements

JEL classification: C72; D02; H41; Q54

\section{Introduction}

We augment the standard cartel formation game from non-cooperative coalition theory, often applied in the context of international environmental agreements (IEAs) (cf. Hoel, 1992; Carraro and Siniscalco, 1993; Barrett, 1994), with the possibility that singletons support coalition formation without becoming coalition members themselves. Rather, their support takes the form of a monetary transfer to the coalition. The transfer is paid in order to incentivize other agents to participate in a coalition that coordinates their members'

\footnotetext{
*We thank Stefan Ambec, Lorenzo Cerda Planas, Harold Houba, and Gerard van der Meijden, as well as seminar participants at CESifo, EAERE 2015, FAERE 2015, and SURED 2016 for comments and discussion. We acknowledge financial support from FP7-IDEAS-ERC Grant No. 269788.

'Department of Spatial Economics, Vrije Universiteit Amsterdam.

*Department of Social Sciences, Wageningen University.

${ }^{\S}$ Department of Spatial Economics, Vrije Universiteit Amsterdam, and Tinbergen Institute.
} 
actions to provide a public good, for example abatement of emissions. In the model, a larger coalition implies an increase in the amount of public goods provided, which is the prime incentive to provide transfers to the coalition. We refer to the agents who make a payment to the coalition as supporters. We show that there exist equilibria (that is, stable coalition structures) with a strictly positive number of supporters. The existence of supporters increases the size of stable coalitions, the contributions to the public good, and the payoffs to each of three types of agents: members, supporters and free-riders.

A standard application of the model used in the current paper is the formation of IEAs for climate change mitigation (Finus, 2003). Up till now, countries' cooperation to reduce greenhouse gas emissions has taken off slowly, with the Kyoto Protocol as the prime example and the Paris Agreement as the latest agreement to enter into force. To the best of our knowledge, however, these agreements - as well as other IEAs—have not (yet) seen financial support by non-participating countries. One possible reason is that many IEAs appear to be void in the sense that ratifying countries would have reached the targets set in the agreement also non-cooperatively (Finus and Tjøtta, 2003; Böhringer and Vogt, 2004). If this feature of IEAs is common knowledge among the agents, there is no incentive for support. Yet, when cooperating countries would manage to agree on actions that go beyond non-cooperative behavior, our paper demonstrates that augmenting IEAs with the option of support would significantly improve the prospects for wider cooperation.

This result is substantiated by the following two other examples of public goods. The first example is given by peace-keeping missions. Typically a small core group of countries (the coalition) joins to lead the mission in terms of sending troops and equipment (Bove and Elia, 2011), with a set of other countries supporting this coalition financially (Khanna et al., 1998; Shimizu and Sandler, 2002). In the case of UN-missions, this support is partly formalized trough a sharing rule for peace-keeping costs, although countries may opt out. In the case of non-UN missions, regional aspects and private incentives affect whether countries send troops or choose to provide financial support (Bobrow and Boyer, 1997; Gaibulloev et al., 2009). The second example is the occurrence of private charitable giving by donors to volunteer organizations. Typically, such charities use part of their gift income to expand their activities by recruiting additional volunteers, and possibly compensating them for any costs incurred. Contrary to donating money, volunteers donate their time to work for the charity. Volunteer organizations therefore rely on two types of charitable activities: a group of volunteers (i.e. the coalition) contribute their time to provide the charitable good, while a set of donors supports the charity financially. ${ }^{1}$ The

\footnotetext{
${ }^{1}$ Although the two types may partially overlap within households or individuals (Freeman, 1997), charitable giving and volunteering are generally seen as substitutes rather than complements (Feldman, 2010;
} 
common denominator in both examples is that agents can choose from different options to contribute to the public good. Our paper offers a general model of public goods provision with support from singletons.

The model that we use is a standard cartel formation game which features simultaneous membership decisions, a single coalition, and open membership. We augment this model with the option that (a set of) singletons support the coalition. We develop the model as a sequential game where agents first decide to become supporters or not. Subsequently, the remaining players decide to become members or not, given the support that is offered to the coalition. Finally, given the coalition structure, each agent determines his contribution to the public good. At this stage the coalition members coordinate their actions; essentially they act as a single player. We solve the game backwards and determine the Subgame Perfect Nash equilibria (SPNE) in support and membership strategies, as well as the associated level of supporting transfer and total abatement.

We show that, under mild conditions on the costs and benefits of contributing to the public good, there exist equilbria with support. For two common model specifications we show that equilibria with support significantly improve equilibrium IEA sizes and increase welfare. Although our model is a general public goods game, we frame it as an international climate policy game for concreteness. Thus, in the remainder of the paper, we refer to the agents as countries, to the coalition as the IEA, and to the public good as abatement.

Our paper is inspired by a classic paper on IEA formation by Carraro and Siniscalco (1993), who analyze four types of commitment to expand coalitions beyond what is feasible non-cooperatively. One of these (see their Proposition 5 on external commitment) is the possibility that a subset of singletons makes transfers to the coalition, thereby inducing the remaining singletons to join the coalition. This resembles the setting of the current paper, but there are three main differences. First, Carraro and Siniscalco (1993) rule out the existence of free-riders; countries are either member or supporter. In the current paper, we endogenize the number of members, allowing for the existence of free-riders in equilibrium. Second, we derive more plausible stability conditions that are based on a sequential game. This allows us to check, for instance, whether or not free-riders have an incentive to become members or to support the IEA, taking into account possible effects on other countries' behavior in subsequent stages of the model. The third difference relates to commitment. Finus (2003) correctly observes that: “... commitment is not compatible with the notion of self-enforcing IEAs. In fact, assuming enough commitment, any problem of cooperation can trivially be solved"; see also Carraro and Siniscalco (1993), p.315. Different from Carraro and Siniscalco (1993) our model does not require any commitment that is exogenously

Bauer et al., 2013). 
imposed on the players. In our model players become supporters or IEA members driven by payoff-maximization. Note however that we do assume a self-imposed commitment which is standard in non-cooperative IEA models: becoming a supporter or an IEA member implies a commitment to comply with, respectively, transfer obligations and abatement.

Carraro and Siniscalco (1993) find that commitment is required for equilibria with support, without further examining the sensitivity of this result with respect to the model setup. Subsequent research has taken this result for granted, so that further analysis on external support has been largely neglected in the literature (as opposed to the other types of commitment analyzed by Carraro and Siniscalco (1993)). One exception is Carraro et al. (2006) who analyze the role of various types of transfers in stabilizing IEAs using simulations. One of the transfers they consider is a direct transfer from a free-rider to another free-rider in order to 'bribe' him to join the coalition. This transfer is reminiscent of the supporting transfers in our model, but we analyze such transfers analytically and in a richer setting. The lack of attention for external support is somewhat surprising since the potential impact of support on cooperation appears to be substantial. Our paper therefore contributes to the policy discussion on the design, or architecture, of IEAs (Aldy and Stavins, 2009). Specifically, the option of support allows contributions to abatement by countries in differentiated ways, a central tenet in climate change negotiations. Differentiated participation is usually connected with broadening participation as well as broadening the scope for agreement negotiations (Olmstead and Stavins, 2012), a premise that we will see confirmed in this paper.

In the next section we introduce the model. In Section 3 we present our existence result for equilibria with support. In Section 4 we apply this result to two common model specifications and we illustrate the impacts of support on equilibrium IEA size and welfare. In Section 5 we discuss a modification of our game and its relation to Carraro and Siniscalco (1993). Conclusions are provided in Section 6.

\section{An IEA model with supporters}

The standard model of IEA formation is a cartel formation game with two stages where countries choose to sign up as coalition members in the first stage. In the second stage, an abatement game is played in which each country chooses its abatement of greenhouse gas emissions. In this game, coalition members act as a single agent by coordinating their abatement levels so as to maximize joint payoffs, which is the default assumption in the literature on IEAs. Employing this assumption, Samuelson's rule applies since abatement is a public good, and members equate individual marginal abatement costs to the sum of 
members' marginal abatement benefits (Barrett, 1994).

Our focus in this paper is to explore the potential impact that allowing for support can have to increase abatement of greenhouse gas emissions and to overcome the inefficiencies inherent in the free-rider problem. We will therefore pay attention to the question how a climate agency could make the best use of the potential impact of support on IEA formation. One can think of the agency as an international body that coordinates climate policy making. In this paper, however, since we do not model the process of negotiations explicitly, a better interpretation is to think of the agency as a facilitator of such a process that generates two contracts: (i) a support contract that countries can sign at stage 1 which commits its signatories to coordinate their transfer with all supporters; and (ii) an IEA contract that countries can sign at stage 3 which commits its signatories to coordinate their abatement with all IEA members. In the remaining two stages, stage 2 and 4, coordinated actions by supporters and members lead to, respectively, particular levels of supporting transfers and abatement. The climate agency plays a key role in the selection of the transfer payment by preparing a transfer proposal that supporters can accept or reject. It is natural to assume that the agency can condition the transfer it suggests on the number of supporters. In other words, the agency's proposal is a transfer scheme. In our game, every country is aware of the details of the transfer scheme, further introduced below.

The key issue that the climate agency faces is to incentivize IEA membership through supporting transfers while, at the same time, it must be attractive for countries to provide support. The former asks for large transfers while the latter must seek to keep transfers low. Note that we do not model the climate agency as a player in our game. Rather, we consider an agency that sets the rules of the game, through the preparation of the two contracts.

Our game has four stages. ${ }^{2}$

1. All countries independently and simultaneously choose to become a supporter or not.

2. The supporters choose their transfer payment to the IEA by accepting or rejecting the agency's proposal.

3. The remaining countries independently and simultaneously choose to become a member or not. This leads to a partition of the set of countries into three subsets: supporters, members and free-riders.

\footnotetext{
${ }^{2}$ Note that our 4-stage model can be generalized to a setting more similar to Carraro and Siniscalco (1993), where an IEA has already formed and subsequently supporters provide a transfer to broaden the coalition. This setting requires a few changes in terminology but the analysis remains unchanged. For instance, the set of "all countries" in stage 1 would refer to the singletons that are not (yet) in the IEA, while the membership choice in stage 3 refers to singletons that would 'join' the initial members of the IEA. Such a generalization would not affect our results.
} 
4. All countries choose their abatement level. Like in the standard IEA game, members coordinate their abatement levels and all other countries (supporters and free-riders) act as singletons. At this stage all transfers are paid and all payoffs are determined.

We proceed by introducing the model more formally.

\subsection{The game}

Consider a set $N=\{1,2, \ldots, n\}$ consisting of $n$ symmetric countries. In stage 1 , each country decides to become a supporter or not. We denote this decision by $\sigma_{i} \in\{0,1\} \forall i \in N$ with the interpretation that when $\sigma_{i}=1$ is chosen, $i$ will become a supporter but otherwise not. The outcome of stage 1 is a set $S=\left\{i: \sigma_{i}=1\right\}$, which comprises all supporters. The number of supporters is denoted $s=|S|$.

In stage 2, the set of supporters $S$ decides whether or not to make the proposed transfer payment $t(s)$ to the IEA. Here we assume $t(s)$ is not negotiable, but it can be rejected. The strategy set at this stage is therefore $\{0, t(s)\}$. With the option to reject $t(s)$ we take into account that supporters' contributions are voluntary, i.e. the set of $s$ supporters will accept a payment $t(s)$ only if they are not worse off compared to not making a transfer. A natural feature of a model with ex ante symmetric countries ${ }^{3}$ is that both the transfers by supporters and the distribution of the transfer payment among members is based on equal sharing (Yi, 1997). Hence, the transfer by each individual supporter equals $t(s) / s$. Note that in Section 5.1, we will drop the role of the agency in proposing a transfer scheme and, instead, let the set of supporters choose a transfer that maximizes their joint payoff.

In stage 3 , given $t$, the remaining $n-s$ countries decide whether to join the IEA or not. We denote this decision by $\mu_{i} \in\{0,1\} \forall i \in N$ with the interpretation that when $\mu_{i}=1$ is chosen, $i$ will become a member but otherwise not. The outcome of stage 3 is a set $M=\left\{i: \mu_{i}=1\right\}$, which comprises all members. The number of members is denoted $m=|M|$. At the end of stage 3 the set of agents is partitioned into three subsets of agents, $\{S, M, F\}$, where the set $F=N \backslash\{S \cup M\}$ comprises the free-riders.

In stage 4 , given $s$ and $m$, all countries determine their abatement levels $q_{i}$. Free-riders and supporters choose abatement to maximize their individual payoffs. IEA members coordinate their abatement decisions to maximize joint payoffs. All individual abatement levels add up to a global abatement level $q=\sum_{j \in N} q_{j}$. Hence, the abatament game in this stage is an additive public goods game.

\footnotetext{
${ }^{3}$ Despite countries being symmetric ex ante, they may receive different payoffs ex post due to their decisions in stages 1 and 3.
} 
Payoffs are based on the benefits $b(q)$ and costs $c\left(q_{i}\right)$ of abatement and on the transfer payment $t$. The benefit function $b(q)$ is increasing and concave while the cost function $c\left(q_{i}\right)$ is increasing and strictly convex. Benefits depend on total abatement, while costs depend only on individual abatement.

Free-riders obtain

$$
w_{i}(q)=b(q)-c\left(q_{i}\right) \text { for all } i \in F
$$

Supporters obtain

$$
w_{i}(q)=b(q)-c\left(q_{i}\right)-\frac{t(s)}{s} \text { for all } i \in S .
$$

Members obtain

$$
w_{i}(q)=b(q)-c\left(q_{i}\right)+\frac{t(s)}{m} \text { for all } i \in M .
$$

\subsection{Solving the game: IEA membership}

We apply SPNE and solve the game backwards. ${ }^{4}$

In stage 4, given symmetric agents, equilibrium abatements are determined from the best response functions of IEA members and free-riders

$$
q_{i}=\arg \max _{\tilde{q}_{i}} m \cdot b\left(\sum_{j \neq i} q_{j}+\tilde{q}_{i}\right)-c\left(\tilde{q}_{i}\right), \quad i \in M
$$

and

$$
q_{i}=\arg \max _{\tilde{q}_{i}} b\left(\sum_{j \neq i} q_{j}+\tilde{q}_{i}\right)-c\left(\tilde{q}_{i}\right), \quad i \in N \backslash M
$$

Each country takes abatement by all other countries as given and chooses its individual abatement either to maximize the net benefits to the IEA (IEA members), or to maximize individual net benefits (all other countries).

Under the assumption that the abatement game at this stage has a unique equilibrium, each country's individual equilibrium abatement can be written as function of the number of IEA members $m .{ }^{5}$ Define $\bar{q}(m)$ and $\underline{q}(m)$ as the equilibrium abatement levels of members

\footnotetext{
${ }^{4}$ Alternative equilibrium concepts may be used, such as those based on farsightedness (cf. Diamantoudi and Sartzetakis, 2015), but we stick to the standard approach.

${ }^{5}$ This will be guaranteed if the corresponding transboundary pollution game is smooth and regular and the pollution is uniformly distributed. See Folmer and von Mouche (2000, Proposition 3).
} 
and singletons, respectively. $\bar{q}(m)$ and $q(m)$ are determined by solving the system of best responses given by (4) and (5). When $\underline{q}(m)$ is decreasing in $m$, leakage occurs. That is, part of the cooperative abatement by the IEA members is offset through decreased abatement by singletons.

Let $\kappa(m)$ denote the welfare of country $i$ obtained from abatement when country $i$ and $m-1$ other countries are in the IEA. Similarly, let $\lambda(m)$ denote country $i$ 's welfare obtained from abatement if $i$ is not in the IEA formed by $m$ other agents. By convexity of $c\left(q_{i}\right)$ we have $q(m) \leq \bar{q}(m)$. A member's payoff, excluding transfers, $\kappa(m)$ and a singleton's payoff $\lambda(m)$ are defined as follows:

$$
\begin{array}{ll}
\kappa(m)=b(m \cdot \bar{q}(m)+(n-m) \cdot \underline{q}(m))-c(\bar{q}(m)) & \forall i \in M, \\
\lambda(m)=b(m \cdot \bar{q}(m)+(n-m) \cdot \underline{q}(m))-c(\underline{q}(m)) & \forall i \in N \backslash M .
\end{array}
$$

Due to the public good characteristic of abatement the payoffs differ only on the cost side and we have $\kappa(m) \leq \lambda(m)$ for any $m$.

Turning to stage 3, definitions (6) and (7) can be used to spell out the equilibrium conditions for this stage. A Nash equilibrium in membership strategies implies that no member would like to deviate from $\mu=1$, and also no free-rider would like to deviate from $\mu=0$. We can state two conditions:

$$
\begin{aligned}
\kappa(m)+\frac{t(s)}{m} & \geq \lambda(m-1), \\
\lambda(m) \quad & \geq \kappa(m+1)+\frac{t(s)}{m+1} .
\end{aligned}
$$

Conditions (8) and (9) are called internal and external stability (D'Aspremont et al., 1983) of the IEA. An IEA that satisfies both conditions is called stable. It is easy to see from (8) that a larger transfer supports internal stability but from (9) we see that a larger transfer undermines external stability.

\subsection{Solving the game: Support}

In stage 2, the set of supporters $S$ decides whether or not a given transfer $t(s)$, as proposed by the agency, will be paid to incentivize other countries to participate in the IEA. To spell out the equilibrium condition for this stage we need to compare the payoffs obtained by the supporters when they accept or reject the proposal $t(s)$ by the agency. If they accept, the transfer $t(s)$ will implement a stable coalition at stage 3 . So we can invoke conditions (8) and (9). Rearranging (8) gives the required transfer to internally stabilize an IEA of size $m$ : 
$t \geq m \cdot(\lambda(m-1)-\kappa(m))$. We can therefore identify all $m$ such that (8) holds for $t$. Of these internally stable coalitions we can identify the subset that is also externally stable such that (9) is satisfied. We denote the set of internally and externally stable coalitions under transfer $t$ by $\mathcal{M}(t) . \mathcal{M}(t)$ is non-empty. To see this, notice that for given $t$, if external stability (9) is violated for $m$, then (8) holds for $m+1$ and we can apply this argument repeatedly until (9) holds or the set of potential members is exhausted, $m=n-s$. While $\mathcal{M}(t)$ is non-empty, we cannot be sure that $\mathcal{M}(t)$ is a unit set. In what follows, with slight abuse of notation, we refer to the elements of $\mathcal{N}(t(s))$ as $m(t(s))$.

Let $\hat{m}$ be the largest $m$ such that (8) and (9) are satisfied for $t=0$. Formally, $\hat{m} \in \mathcal{M}(0)$. In other words, $\hat{m}$ is the largest IEA without support. Then, for an equilibrium at stage 2 , we require.

$$
\lambda(m(t(s)))-\frac{t(s)}{s} \geq \lambda(\hat{m})
$$

Hence, in an equilibrium with support, if condition (10) holds, it is the best option for the set of supporters to pay the transfer as proposed by the agency.

In stage 1 , all countries have to decide whether to become a supporter or not. This decision will depend on the transfer that has to be paid, with how many other supporters the transfer can be shared and, of course, the impact of the transfer on IEA formation and the resulting global abatement.

To spell out equilibrium conditions for this stage, we need to consider two cases. In an equilibrium of the subgame following to stage 1 , any country that is not a supporter will either become an IEA member or a free-rider in stage 3. A Nash equilibrium in support strategies implies that no supporter deviates from $\sigma_{i}=1$ and no other country deviates from $\sigma_{i}=0$, taking into account these stage 3 decisions. Hence, the following internal stability conditions must hold. If country $i$ would become an IEA member in the subgame following its deviation, its member payoff in the subgame with $s-1$ supporters should be lower than its supporter payoff in the subgame with $s$ supporters:

$$
\lambda(m(t(s)))-\frac{t(s)}{s} \geq \kappa(m(t(s-1)))+\frac{t(s-1)}{m(t(s-1))} .
$$

If country $i$ would become a free-rider in the subgame following its deviation, its free-rider payoff in the subgame with $s-1$ supporters should be lower than its supporter payoff in the subgame with $s$ supporters:

$$
\lambda(m(t(s)))-\frac{t(s)}{s} \geq \lambda(m(t(s-1)))
$$


Conditions (11) and (12) say, respectively, that a supporter's payoff must be at least as large as the payoff obtained as a member or a free-rider when an IEA of size $m(t(s-1))$ prevails. In other words, we have two internal stability conditions for $S$. These conditions say that a country will become a supporter if the associated payoff is at least as large as what can be obtained in a subgame that follows the choice of $\sigma=0$.

Similarly, we have two external stability conditions since players who do not become supporters may or may not become IEA members in the subgame that follows. For a non-supporting country that would become a free-rider, its supporter payoff in the subgame with $s+1$ supporters should be lower than its free-rider payoff in the subgame with $s$ supporters:

$$
\lambda(m(t(s))) \geq \lambda(m(t(s+1)))-\frac{t(s+1)}{s+1} .
$$

For a non-supporting country that would become an IEA member, its supporter payoff in the subgame with $s+1$ supporters should be lower than its member payoff in the subgame with $s$ supporters:

$$
\kappa(m(t(s)))+\frac{t(s)}{m(t(s))} \geq \lambda(m(t(s+1)))-\frac{t(s+1)}{s+1} .
$$

In other words, we have two external stability conditions for $S$. These conditions say that a country will not become a supporter if the associated payoff as a free-rider or a member is at least as large as what can be obtained in a subgame with $s+1$ supporters that follows the choice of $\sigma=1$.

\section{Existence of equilibria with supporters}

In the previous section we have derived the conditions that an SPNE must satisfy. For the idea of support to have any relevance we must now show that equilibria with support exist. This is not trivial. For instance, using the quadratic-quadratic model specification as introduced in Section 4.2 below, there exist parameter combinations for which there is no equilibrium with support, as illustrated in Figure 3. The bottleneck appears to be that the benefits from an enlarged IEA to supporters are not sufficient to make up for the payment they have to make. In other words, to find equilibria we have to show, among other things, under which conditions positive spillovers to the supporters are (weakly) larger than the required payment to stabilize an IEA.

Equilibria with supporters can be identified by the numbers of supporters and members 
as established in stages 1 and 3. The size of the set of supporters, $s$, and the size of the set of members, $m$, are associated with a unique level of the transfer $t(s)$ in stage 2 as well as abatement $q(m)$ in stage 4, respectively.

Equilibria are established by checking - given $t(s)$-whether there exists $m(t(s))$ that satisfies a set of conditions. These include internal and external stability of the IEA (8) and (9), the constraint on support (10), and internal and external stability of the set of supporters (11)-(14).

In light of (8), we define the minimum transfer needed to internally stabilize an IEA of size $m>\hat{m}$ as

$$
\tau(m)=m \cdot(\lambda(m-1)-\kappa(m))
$$

We define $\tau(\hat{m})=0$. A natural assumption on behalf of the climate agency is that the proposed $t(s)$ will not exceed $\tau(m)$ so that $t(s)=\tau(m)$. Hence, if the agency aims at having $m$ members, the transfer will be set equal to $\tau(m)$.

We now prove existence of equilibria with support, provided $n-m$ is sufficiently large. This condition is not very demanding, which we demonstrate in Section 4 with results for two common specifications of the game.

Proposition 1. If for some $m^{*}>\hat{m}$ it holds that $n-m^{*} \geq \tau\left(m^{*}\right) /\left(\lambda\left(m^{*}\right)-\lambda(\hat{m})\right)$, then there exist (one or more) SPNE $\left(s^{*}, m^{*}\right)$ with $0<s^{*} \leq n-m^{*}$.

Proof. To establish existence of equilibria, we need to verify conditions (8)-(14) for some function $t^{*}(s)$ and integers $s^{*}$ and $m^{*}$. Consider that the agency is seeking to establish an IEA of some size $m^{*}>\hat{m}$ that would not be stable without support so that $\tau\left(m^{*}\right)>0$. A sufficient condition for such $m^{*}$ to exist is that $\hat{m}<n$, since otherwise the grand coalition would form without support.

Next, we introduce $t^{*}(s)$ and $s^{*}>0$ that we will use to satisfy the abovementioned conditions for the targeted IEA size $m^{*}$. Consider the largest possible set of supporters $s^{*}=n-m^{*}$ and the following transfer scheme

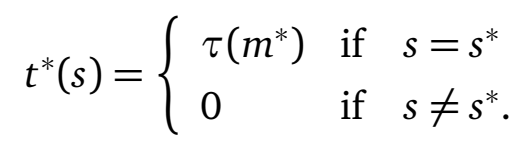

Using this transfer scheme, with $\tau\left(m^{*}\right)=m^{*}\left(\lambda\left(m^{*}-1\right)-\kappa\left(m^{*}\right)\right)$, the agency is targeting IEA size $m^{*}$ with support from $n-m^{*}$ supporters.

We proceed to verify whether conditions (8)-(14) are satisfied by $t^{*}(s), s^{*}$, and $m^{*}$. Conditions (8) and (9) hold by construction as $m^{*} \in \mathcal{M}\left(t^{*}\left(s^{*}\right)\right)$, while condition (13) does 
not apply in absence of free-riders (since $n=m^{*}+s^{*}$ ). We proceed with the remaining conditions, which we rewrite using (16):

$$
\begin{aligned}
& \lambda\left(m^{*}\right)-\frac{m^{*}\left(\lambda\left(m^{*}-1\right)-\kappa\left(m^{*}\right)\right)}{n-m^{*}} \geq \lambda(\hat{m}) . \\
& \lambda\left(m^{*}\right)-\frac{m^{*}\left(\lambda\left(m^{*}-1\right)-\kappa\left(m^{*}\right)\right)}{n-m^{*}} \geq \kappa(\hat{m}) \\
& \lambda\left(m^{*}\right)-\frac{m^{*}\left(\lambda\left(m^{*}-1\right)-\kappa\left(m^{*}\right)\right)}{n-m^{*}} \geq \lambda(\hat{m}) \\
& \kappa\left(m^{*}\right)+\frac{m^{*}\left(\lambda\left(m^{*}-1\right)-\kappa\left(m^{*}\right)\right)}{m^{*}} \geq \lambda(\hat{m}) .
\end{aligned}
$$

Conditions (11') and (14') can be ignored. First, it follows directly from (6) and (7) that $\kappa(\hat{m}) \leq \lambda(\hat{m})$ and therefore (11') is implied by (12'). Second, (14') simplifies to $\lambda\left(m^{*}-1\right) \geq \lambda(\hat{m})$ which is satisfied since we have $m^{*}-1 \geq \hat{m}$ and we know that $\lambda(m)$ is increasing in $m$ (Weikard et al., 2006, Proposition 3).

The two remaining conditions (10') and (12') are identical and establish the existence of an SPNE $\left(s^{*}, m^{*}\right)$ with $s^{*}=n-m^{*}$ and $m^{*}>\hat{m}$ whenever

$$
n-m^{*} \geq \tau\left(m^{*}\right) /\left(\lambda\left(m^{*}\right)-\lambda(\hat{m})\right)
$$

This establishes existence of an $\operatorname{SPNE}\left(s^{*}, m^{*}\right)$ with $s^{*}=n-m^{*}$. Additional SPNE with $s^{*}<n-m^{*}$ are possible whenever

$$
s^{*} \geq \tau\left(m^{*}\right) /\left(\lambda\left(m^{*}\right)-\lambda(\hat{m})\right)
$$

and condition (13) holds since now we have free-riders $\left(n>m^{*}+s^{*}\right)$. Condition (13) simplifies to $\lambda\left(m^{*}\right) \geq \lambda(\hat{m})$ which is implied by $\left(14^{\prime}\right)$.

The idea of our proof to show the existence of support equilibria rests on a transfer scheme that makes paying support as attractive as possible. The transfer $\tau(m)$ is the smallest transfer that internally stabilizes an IEA of size $m$. Furthermore, the transfer scheme $t^{*}(s)$ is designed to discourage deviations at stage 1 . Any deviation would give the no-support payoff to all countries, including the deviators. For that reason we refer to transfer scheme (16) as the grim transfer scheme. If spillovers from an enlarged IEA are large enough to make support worthwhile at all, then the grim transfer scheme that we employ will be able to implement such an IEA enlargement via a transfer that is beneficial to supporters as well. Furthermore, it is clear from the construction of the grim transfer scheme that any equilibrium $\left(s^{*}, m^{*}\right)$ that prevails under some transfer scheme $t(s)$ will 
also be an equilibrium under the grim transfer scheme. ${ }^{6}$

The climate agency is certainly not bound to use transfer scheme $t^{*}(s)$, which we use here only to prove existence of support equilibria. Instead, it can use alternative schemes that have more attractive features. One such feature, violated by $t^{*}(s)$, is monotonicity of $t(s)$ in $s$. Intuitively it makes sense that transfers increase as the number of supporters increase. The equilibria that result from any alternative transfer scheme can be easily checked numerically.

Clearly, paying support is a burden that is easier to carry if the number of supporters is large. The size of the spillovers, i.e. the additional benefits from abatement as the IEA is enlarged through support, limits what a supporter would be willing to pay. Therefore it is often, but not always, possible to find large IEAs if there are sufficiently many countries that are potential supporters. This is the essence of condition (17). The next section illustrates this result. Notice that support may fail, i.e. condition (17) is violated for all $n$, if leakage is sufficiently strong. To see this, recall that the function $\lambda(m)$ is defined for a game with a given number of players $n$. When considering (17) to determine the number of potential supporters, $n-m$, an increase in $n$ increases the RHS. If there is leakage, $\lambda(m)$ will fall as $n$ increases. In Section 4.2 we will provide an example where leakage is too strong to allow for support equilibria. Turning this argument around, we can say that if there is no leakage, i.e. $\lambda(m)$ is non-decreasing in $n$, we will always find support equilibria for sufficiently large $n$. The most prominent example of an abatement game without leakage is a game with linear benefits and quadratic costs of abatement. To this case we turn next and we will show that large IEAs can be stabilized with support.

\section{Application to two common model specificiations}

In this section we apply our existence result to two common model specifications and we illustrate how support can improve equilibrium IEA sizes and increase welfare. The first is a specification with linear benefits and quadratic costs, while in the second we consider quadratic benefits and quadratic costs. Similar specifications have been used since Barrett (1994) by e.g. Botteon and Carraro (1997), Barrett (2006), Diamantoudi and Sartzetakis (2006), Rubio and Ulph (2006), and Finus and Maus (2008).

\footnotetext{
${ }^{6}$ To see why any equilibrium $\left(s^{*}, m^{*}\right)$ that prevails under some transfer scheme $t(s)$ will also be an equilibrium under the grim transfer scheme, note that to establish existence of equilibria we need to verify conditions (8), (9), and (10)-(14) for some $t^{*}(s), s^{*}$, and $m^{*}$. Since $\left(s^{*}, m^{*}\right)$ is an equilbrium, we have $t\left(s^{*}\right)=\tau\left(m^{*}\right)$. Whether conditions (8), (9), and (10) are satisfied depends only on $m^{*}, s^{*}$, and $t\left(s^{*}\right)$ but is otherwise independent of the transfer scheme. For the remaining conditions (11)-(14) the LHS of each of these is again independent of the transfer scheme, while the RHS is minimized under transfer scheme $t^{*}(s)$.
} 


\subsection{Linear-quadratic}

We start with the linear-quadratic specification with benefit function $b(q)=\beta q$ and cost function $c\left(q_{i}\right)=\frac{1}{2} \gamma q_{i}^{2}$. The linear-quadratic specification implies that each country has a dominant abatement strategy. Using (4) and (5) we have $\bar{q}(m)=m \beta / \gamma$ and $q(m)=\beta / \gamma$, which we use to write $\kappa(m), \lambda(m)$, and $\tau(m)$ as follows:

$$
\begin{aligned}
\kappa(m) & =\frac{\beta^{2}}{\gamma}\left(\frac{1}{2} m^{2}+n-m\right), \\
\lambda(m) & =\frac{\beta^{2}}{\gamma}\left(m^{2}+n-m-\frac{1}{2}\right), \\
\tau(m) & =\frac{\beta^{2}}{\gamma} m\left(\frac{1}{2} m^{2}-2 m+\frac{3}{2}\right) .
\end{aligned}
$$

Next, consider the possibility of supporting transfers. Without support, a well-known results is that $\hat{m}=3$ whenever $n \geq 3$ (Barrett, 1994, Proposition 2). Using (20) and (21) and plugging in $\hat{m}=3$, the existence condition (17) for the linear-quadratic case is

$$
n-m^{*} \geq \frac{m^{*}\left(\left(m^{*}\right)^{2}-4 m^{*}+3\right)}{2\left(\left(m^{*}\right)^{2}-m^{*}+5\right)} .
$$

Condition (22), whose RHS is converging from below to the asymptote $\left(m^{*}-3\right) / 2+m^{*}$ for $m \rightarrow \infty$, is independent of the model parameters $\beta$ and $\gamma$. We can verify numerically that the smallest equilibrium IEA with support (i.e. $m^{*} \geq \hat{m}+1=4$ ) equals $\left(s^{*}, m^{*}\right)=(1,4)$. Hence, condition (22) is satisfied for any $n \geq 5$.

Now that we have established existence of SPNE for the linear-quadratic case, we proceed to illustrate the wide variety of possible equilibria in Figure 1, for $n=100$. The large shaded triangle contains all equilibria $\left(s^{*}, m^{*}\right)$ that can be implemented by the grim transfer scheme $t^{*}(s)$, see (16). This area is bounded from below since any support equilibrium contains at least $\hat{m}+1=4$ IEA members. It is bounded from above by both the existence condition (17) (left) and by feasibility (i.e. $s^{*} \leq n-m^{*}$ ) given $n=100$ (right). An increase in $n$ will move the feasibility bound outward (and vice versa). Any equilibrium on this feasibility bound consists of IEA members and supporters only, and no free-riders. The highest $m^{*}$ on this bound indicates the largest possible IEA, which comprises about $2 / 3$ of all countries, with the remaining countries acting as supporters, while equilibria below the feasibility bound include free-riders in addition to IEA members and supporters. The linear-quadratic case shows that equilibria with the option of support can achieve significantly higher levels of cooperation, $m^{*} \lesssim \frac{2}{3} n$, than without support, $\hat{m}=3$. 


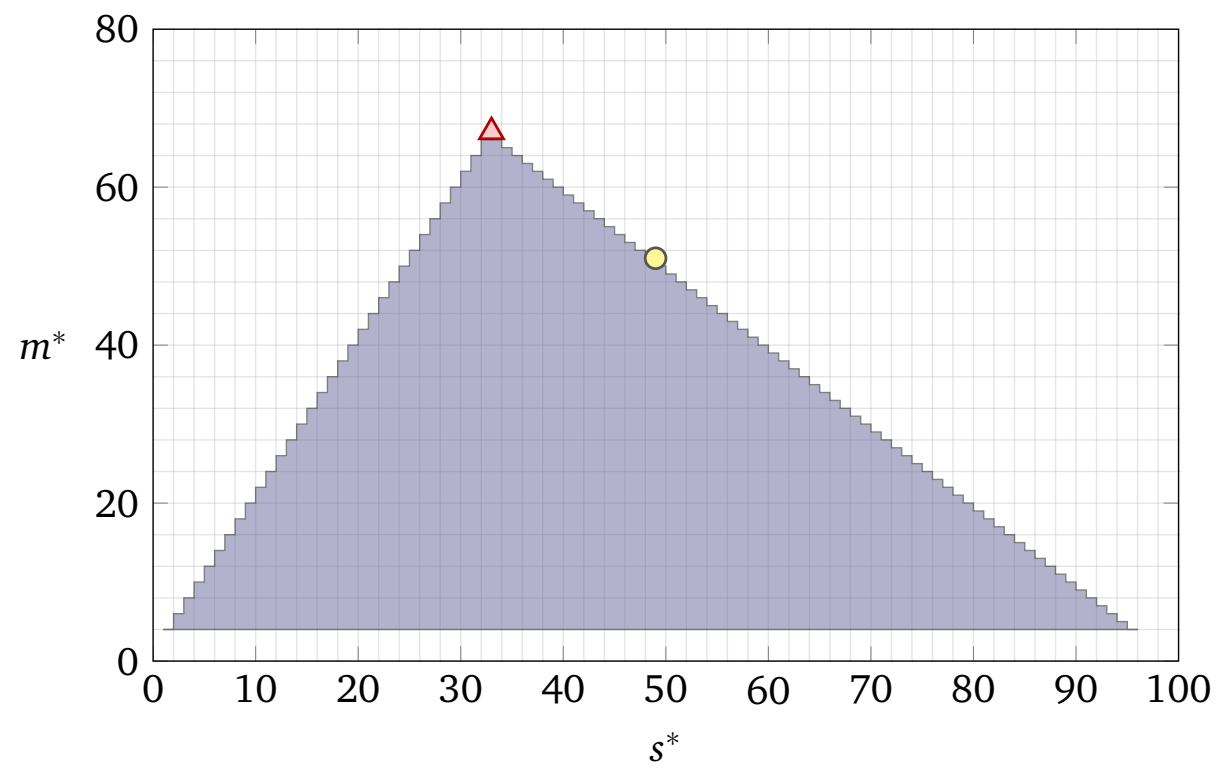

Figure 1: Equilibria $\left(s^{*}, m^{*}\right)$. The shaded area depicts the set of SPNE for the linearquadratic model specification implemented by the grim transfer scheme $t^{*}(s)$ for $n=100$. The red triangle indicates the welfare-maximizing equilibrium, comprising the largest feasible number of members. The yellow circle indicates the equilibrium that maximizes individual supporter payoffs.

Our positive result for support does not hinge on the specific grim transfer scheme $t^{*}(s)$. Although $t^{*}(s)$ is the transfer scheme that can implement the largest possible set of SPNE-see Footnote 6-alternative transfer schemes are able to implement subsets of these SPNE. The selection of a transfer scheme, and thereby the selection of possible equilibria, depends on the objective of the climate agency. A natural assumption is that the agency aims to maximize global welfare, i.e. the sum of payoffs of members, supporters, and free-riders. Since the abatement game in stage 4 is an additive public goods game, this sum depends only on total abatement $q=(m \cdot \bar{q}(m)+(n-m) \cdot q(m))$. Abatement strategies $\bar{q}(m)=m \beta / \gamma$ and $q(m)=\beta / \gamma$ imply that $q$ is increasing in $m$. Therefore, global welfare is maximized when $m^{\star}$ is maximized. ${ }^{7}$ An agency maximizing global welfare would therefore choose $t^{*}(s)$, or any alternative transfer scheme, to implement the highest feasible equilibrium $\left(s^{*}, m^{*}\right)$. Of course, the agency may have an alternative objective like maximization of individual supporter's payoffs. Given $n=100$, these equilibria occur at $\left(s^{*}, m^{*}\right)=(33,67)$ and $\left(s^{*}, m^{*}\right)=(49,51)$, respectively. See Figure 1.

The options to the climate agency are perhaps best illustrated in Figure 2, which displays the welfare effects for individual countries. The figure shows that individual payoffs $w_{i}(q)$

\footnotetext{
${ }^{7}$ For the linear-quadratic specification, $q$ is increasing and convex in $m$. Given $n=100$, total abatement increases from $q=112$ for $m^{*}=\hat{m}+1=4$ to $q=4522$ for $m^{*}=67$.
} 


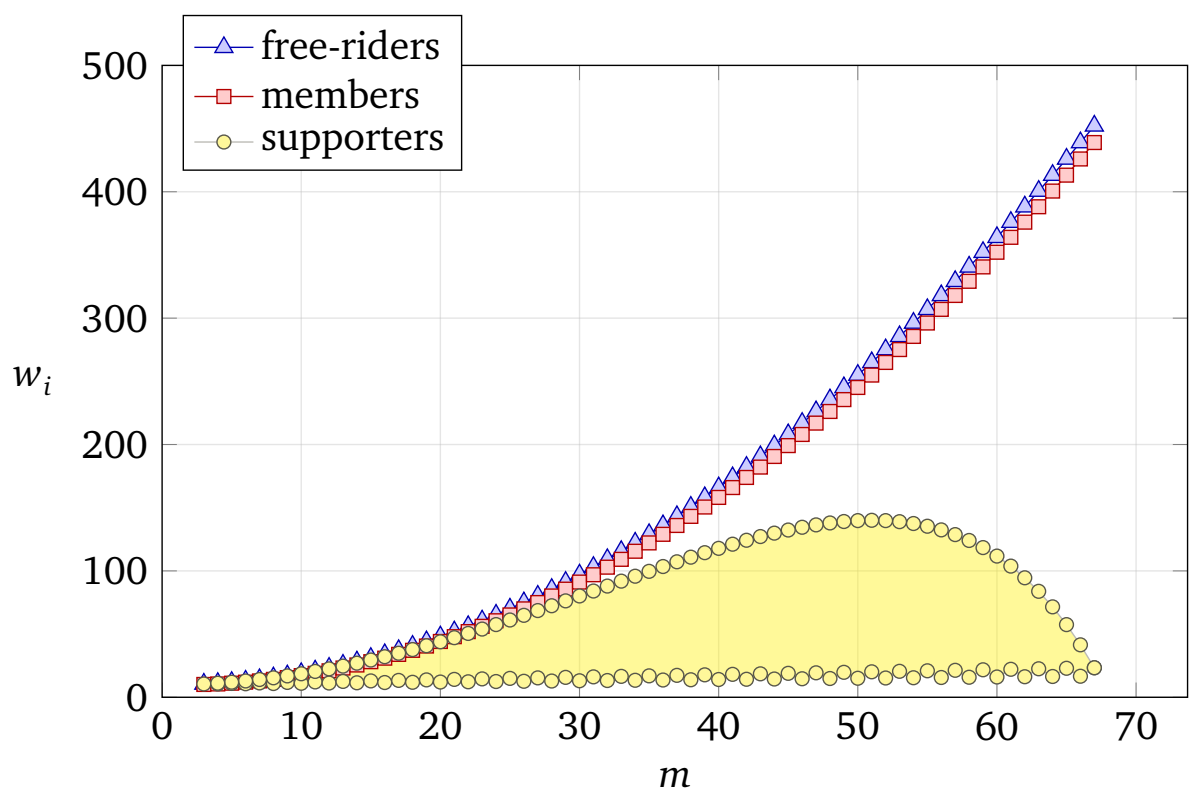

Figure 2: Individual payoffs $w_{i}(q)$ for each of the three types of countries-free-riders, members, and supporters-as a function of $m$, given $n=100$. Supporter payoffs depend on the number of supporters given $m$ as marked by the shaded region. The range of supporters for each $m$ is illustrated in Figure 1.

for each of the three types of countries-free-riders, members, and supporters-is increasing in $m$ up to $m=51$, after which only the maximimum individual supporter payoffs decrease. These maximum payoffs are given by equilbria where $s^{*}=n-m^{*}$, i.e. equilibria at the feasibility bound in Figure 1 where the required transfer is shared by the largest possible number of supporters. Since the feasible range of $s^{*}$ shrinks for higher $m^{*}$, at some point these maximum supporter payoffs have to come down. When the agency's objective is to maximize some function that is positively related to countries' payoffs, Figure 2 shows that (a) all support-equilbria are preferred to the no-support equilibrium with $\hat{m}=3$, (b) larger IEAs are preferred to smaller ones, except when the agency is particularly concerned about supporters' individual payoffs, and (c) for given $m^{*}$, equilibria with more supporters increase individual supporter payoffs while the number of free-riders (who enjoy higher payoffs) is reduced; hence, payoff inequality is reduced.

\subsection{Quadratic-quadratic}

We continue with the quadratic-quadratic specification with benefit function $b(q)=$ $\beta_{1}\left(\beta_{2} q-\frac{1}{2} q^{2}\right)$ and cost function $c\left(q_{i}\right)=\frac{1}{2} \gamma q_{i}^{2}$. The quadratic-quadratic specification differs from linear-quadratic in that it does not feature dominant abatement strategies. Instead, leakage makes that part of the cooperative abatement by the IEA is offset through de- 
creased abatement by supporters and free-riders. From Weikard et al. (2006, Proposition 3), however, we know that leakage is never complete. In other words, total abatement $q$ is non-decreasing in $m$. We could not obtain analytical results for our game with a quadraticquadratic specification, which is not surprising given that simulations are often used for this specification, even without support. We will therefore present simulation results in order to numerically characterize the equilibria for this specification.

In order to simplify the presentation of our results, we will use $n=100$ and set $\beta_{2}=1$. Other values for $\beta_{2}$ do influence abatement and welfare, but do not affect existence of equilibria (cf. Barrett, 1994; Finus and Maus, 2008). We conduct a large number of simulations by varying the two remaining parameters $\beta_{1} \in(0,100]$ and $\gamma \in(0,100]$. For each parameter combination, we check whether we can find any equilibrium $\left(s^{*}, m^{*}\right)$ using the grim transfer scheme $t^{*}(s)$, see Footnote 6 . In Figure 3 we show part of the parameter space to illustrate for which parameter combinations the existence condition (17) is satisfied.

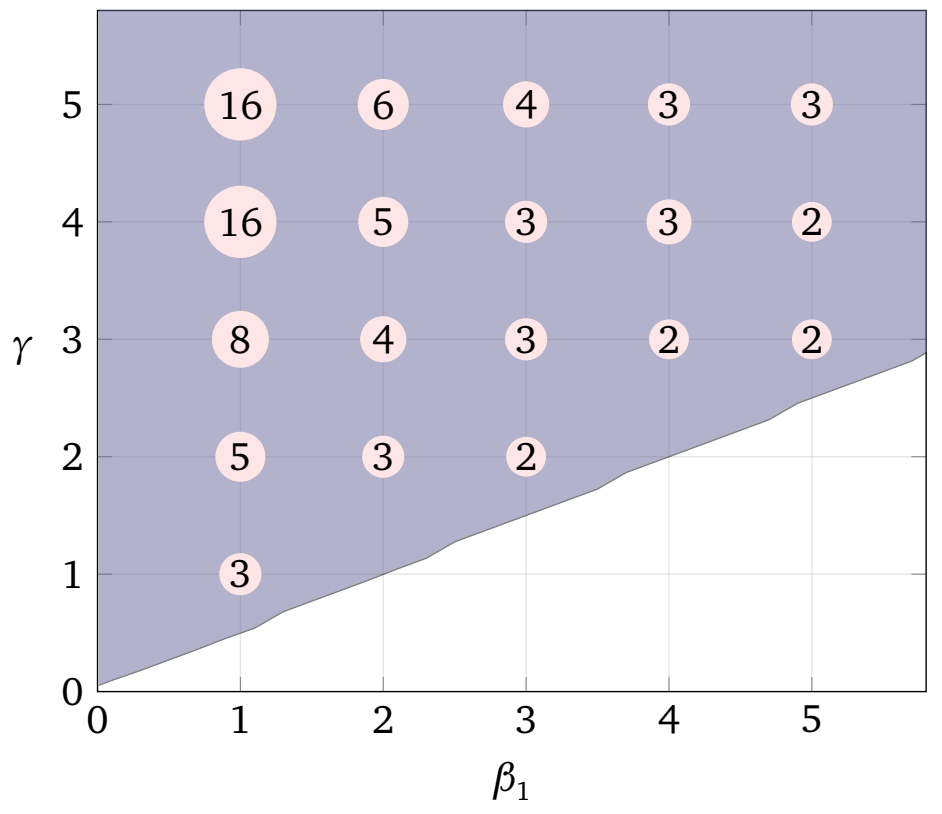

Figure 3: Parameter combinations $\left(\beta_{1}, \gamma\right)$ for which an equilibrium with support exists. The shaded area depicts these combinations for the quadratic-quadratic model specification using $t^{*}(s)$ for $n=100$ and $\beta_{2}=1$. Numbered nodes indicated the size of the largest IEA $m^{*}$ for selected parameter combinations.

A clear trade-off between the two parameters appears. An increasing cost parameter $\gamma$ makes equilibria with support more likely, while an increasing benefit parameter $\beta_{1}$ has a reverse effect. The explanation for this result is that an increasing ratio $\beta_{1} / \gamma$ implies more leakage (cf. Finus, 2003), which makes it harder for supporters to stabilize an IEA. 
Mere existence of support equilibria does not say much about the size of IEAs under such equilibria. To illustrate that relatively large IEAs are possible under the quadratic-quadratic model specification, Figure 3 includes numbered nodes indicating the size of the largest IEA for selected parameter combinations. In the parameter space of Figure 3, these sizes range from $m^{*}=2$ to $m^{*}=16$, which improves significantly on the equilibrium IEA size for the quadratic-quadratic specification without support, which is $\hat{m}=2$ at maximum (Diamantoudi and Sartzetakis, 2006). As $\beta_{1}$ tends to zero, even larger IEAs are possible. For example, we find an IEA of size $m^{*}=79$ when $\beta_{1}=0.01$ and $\gamma=20$.

\section{Discussion}

In this section we discuss a modification of our game and its relation to Carraro and Siniscalco (1993).

\subsection{Support equilibria without a climate agency}

Until now we assumed that a climate agency sets a transfer scheme to support an IEA. In this section we briefly explore an alternative specification of the rules of the game. We drop the assumption of an agency that proposes a transfer scheme $t(s)$, but we maintain the structure of our 4-stage game. Stages 1, 3, and 4 are unchanged. In stage 2, as there is no transfer scheme proposed by the agency, we assume that the set of supporters chooses $t \geq 0$ with the objective of maximizing their joint welfare.

We solve the game backwards as described in Section 2. Clearly, the stability conditions for the IEA, (8) and (9) are maintained. From the analysis of stage 2, however, we obtain a different condition. The analogue to condition (10) is a transfer scheme obtained as a first order condition of the supporters' maximization problem. We have

$$
t^{\prime}(s)=\arg \max _{t}(s \cdot \lambda(m(t))-t)
$$

Turning to stage 1, conditions (11)-(14) apply. Under transfer scheme (23) the set of supporters maximizes their joint welfare, also in subgames that follow after a deviation in stage 1 . As a result, supporters cannot discourage such stage 1 deviations in the same way as the agency can by using, for instance, the grim transfer scheme (16). An implication is that stage 1 deviations are generally more attractive and we expect to find a smaller number of equilibria, if any.

This is confirmed by results for the linear-quadratic model specification. Specifically, 
using transfer scheme (23) we find no equilibrium with support under conditions (8), (9), and (11)-(14).

We do find SPNE, however, when we drop either one or both of (12) and (14). Dropping (12) means that we restrict our attention to equilibria where each individual supporter, upon deviation, would become a member in the subgame with $s-1$ supporters. Since in any equilibrium with support $t(s)=\tau(m)$, condition (8) implies that members have lower payoffs than free-riders. As a result, incentives to deviate are weaker and we have better chances of finding an equilibrium. We find two equilibria: $\left(s^{*}, m^{*}\right) \in\{(4,7),(4,8)\}$, under the condition that each of the four supporters would become an IEA member in stage 3 upon deviviation in stage 1. A similar argument applies when dropping (14). We find two different equilibira: $\left(s^{*}, m^{*}\right) \in\{(1,4),(2,5)\}$, under the condition that each non-supporter that deviates in stage 1 to become a supporter, would have become a free-rider in stage 3 otherwise. Dropping both would give one additional equilibrium $\left(s^{*}, m^{*}\right)=(3,6)$.

Comparing these results to those for the linear-quadratic specification presented in Section 4 shows that, in absence of a climate agency, the supporters are not able to stabilize large IEAs.

\subsection{Comparing our results to Carraro and Siniscalco (1993)}

In Section 1, we described three differences between the setting of this paper and the one by Carraro and Siniscalco (1993). Compared to Carraro and Siniscalco (1993), in this paper we (a) endogenize the number of members; (b) derive stability conditions that are based on a sequential game; and (c) do not require any commitment that is exogenously imposed on the players.

Carraro and Siniscalco (1993) assume that $\lambda(m)>\lambda(\hat{m})$ and $\kappa(m)>\kappa(\hat{m})$ for all $m>\hat{m}$. For any $m>\hat{m}$ they define the transfer like we do in (15):

$$
\kappa(m)+\frac{t}{m}=\lambda(m-1)
$$

Their claim is that if there exists $m>\hat{m}$ such that

$$
\lambda(m)-\frac{t}{n-m} \geq \lambda(\hat{m})
$$

then there is a stable coalition consisting of $m$ members and $n-m$ supporters. Hence, there are no free-riders left, while we allow for free-riders in equilibrium.

They check five stability conditions. Three of these relate to coalition members and these are easity verified: (a) coalition members have no incentive to become free-riders; 
(b) they are better off than coalition members in the equilibrium without support; and (c) they are better off than free-riders in the equilibrium without support. The fourth condition, which holds by (25), is that supporters are better off than free-riders in the equilibrium without support. The final condition is that supporters have no incentive to become coalition members. Carraro and Siniscalco (1993) claim that the relevant condition, implying that "... no additional countries want to join the coalition" is

$$
\lambda(m)-\frac{t}{n-m} \geq \kappa(m+1)
$$

and that this condition follows from the conditions given. First of all, this interpretation is questionable. Note that in their model, without free-riders in equilibrium, the only potential 'additional countries' are supporters. Their payoff is the LHS of (26). The RHS gives the payoff to members if there were one more member, however, without taking into account supporting transfers. It is unclear why a supporting agent would make the suggested comparison. Second, the proof of the claim is flawed. The statement is not correct unless further assumptions are made, for example $\lambda(\hat{m})>\kappa(m+1)$, but this is not easy to justify at all.

Surprisingly, Carraro and Siniscalco (1993) do not check whether coalition members have an incentive to become supporters, nor do they check whether supporters have an incentive to deviate and become a free-rider.

Hence, we think that our approach is more general than Carraro and Siniscalco (1993) and better rooted in the usual game theoretic framework.

\section{Conclusion}

Inspired by Carraro and Siniscalco (1993), we augment the standard cartel formation game from non-cooperative coalition theory with the possibility of support. We show that, under mild conditions on the costs and benefits of abatement, there exist equilibria with support. For two common model specifications we show that equilibria with support significantly improve equilibrium IEA sizes and increase welfare. Our paper therefore highlights an option for IEA design that allows countries to participate in differentiated ways, and with potential to substantially increase (i) cooperation, (ii) abatement of greenhouse gas emissions, and (iii) associated payoffs to all. This option is particularly promising for IEAs for which free-riding incentives are typically large, as is the case for climate change. Hence, we are optimistic about the scope of support as a possible policy tool in the design of IEAs on climate change. 


\section{References}

Aldy, J. and R. Stavins (2009). Post-Kyoto International Climate Policy: Implementing Architectures for Agreement. Cambridge: Cambridge University Press.

Barrett, S. (1994). Self-enforcing international environmental agreements. Oxford Economic Papers 46(S), 878-894.

Barrett, S. (2006). Climate treaties and "breakthrough" technologies. American Economic Review: Papers and Proceedings 96(2), 22-25.

Bauer, T., J. Bredtmann, and C. Schmidt (2013). Time vs. money: The supply of voluntary labor and charitable donations across Europe. European Journal of Political Economy 32(1), 80-94.

Bobrow, D. and M. Boyer (1997). Maintaining system stability contributions to peacekeeping operations. Journal of Conflict Resolution 41(6), 723-748.

Böhringer, C. and C. Vogt (2004). The dismantling of a breakthrough: The Kyoto Protocol as symbolic policy. European Journal of Political Economy 20(3), 597-617.

Botteon, M. and C. Carraro (1997). Burden-sharing and coalition stability in environmental negotiations with asymmetric countries. In C. Carraro (Ed.), International Environmental Negotiations: Strategic Policy Issues. Cheltenham: Edward Elgar.

Bove, V. and L. Elia (2011). Supplying peace: Participation in and troop contribution to peacekeeping missions. Journal of Peace Research 48(6), 699-714.

Carraro, C., J. Eyckmans, and M. Finus (2006). Optimal transfers and participation decisions in international environmental agreements. Review of International Organizations 1(4), 379-396.

Carraro, C. and D. Siniscalco (1993). Strategies for the international protection of the environment. Journal of Public Economics 52(3), 309-328.

D’Aspremont, C., A. Jacquemin, J. Gabszewicz, and J. Weymark (1983). On the stability of collusive price leadership. Canadian Journal of Economics 16(1), 17-25.

Diamantoudi, E. and E. Sartzetakis (2006). Stable international environmental agreements: An analytical approach. Journal of Public Economic Theory 8(2), 247-263. 
Diamantoudi, E. and E. Sartzetakis (2015). International environmental agreements: Coordinated action under foresight. Economic Theory 59(3), 527-546.

Feldman, N. (2010). Time is money: Choosing between charitable activities. American Economic Journal: Economic Policy 2(1), 103-130.

Finus, M. (2003). Stability and design of international environmental agreements: The case of transboundary pollution. In H. Folmer and T. Tietenberg (Eds.), The International Yearbook of Environmental and Resource Economics 2003/2004, pp. 82-158. Cheltenham: Edward Elgar.

Finus, M. and S. Maus (2008). Modesty may pay! Journal of Public Economic Theory 10(5), 801-826.

Finus, M. and S. Tjøtta (2003). The Oslo Protocol on sulfur reduction: The great leap forward? Journal of Public Economics 87(9), 2031-2048.

Folmer, H. and P. von Mouche (2000). Transboundary pollution and international cooperation. In T. Tietenberg and H. Folmer (Eds.), The International Yearbook of Environmental and Resource Economics 2000/2001, pp. 231-266. Cheltenham: Edward Elgar.

Freeman, R. (1997). Working for nothing: The supply of volunteer labor. Journal of Labor Economics 15(1), S140-S166.

Gaibulloev, K., T. Sandler, and H. Shimizu (2009). Demands for UN and non-UN peacekeeping: Nonvoluntary versus voluntary contributions to a public good. Journal of Conflict Resolution 53(6), 827-852.

Hoel, M. (1992). International environment conventions: The case of uniform reductions of emissions. Environmental and Resource Economics 2(2), 141-159.

Khanna, J., T. Sandler, and H. Shimizu (1998). Sharing the financial burden for UN and NATO peacekeeping, 1976-1996. Journal of Conflict Resolution 42(2), 176-195.

Olmstead, S. and R. Stavins (2012). Three key elements of a post-2012 international climate policy architecture. Review of Environmental Economics and Policy 6(1), 65-85.

Rubio, S. and A. Ulph (2006). Self-enforcing international environmental agreements revisited. Oxford Economic Papers 58(2), 233-263.

Shimizu, H. and T. Sandler (2002). Peacekeeping and burden-sharing, 1994-2000. Journal of Peace Research 39(6), 651-668. 
Weikard, H.-P., M. Finus, and J.-C. Altamirano-Cabrera (2006). The impact of surplus sharing on the stability of international climate agreements. Oxford Economic Papers 58(2), 209-232.

Yi, S.-S. (1997). Stable coalition structures with externalities. Games and Economic Behavior 20(2), 201-237. 